Check for updates

The BMJ

Cite this as: BMJ 2021;373:n1427 http://dx.doi.org/10.1136/bmj.n1427 Published: 03 June 2021

\title{
Covid-19: Antimicrobial use was high during first wave despite bacterial co-infections being rare, study finds
}

\section{Elisabeth Mahase}

Measures to prevent the unnecessary use of antimicrobials-such as by restricting prescriptions until a bacterial infection has been confirmed through testing-should be incorporated into covid-19 patient care to help prevent a long term rise of drug resistant infections, researchers have said.

In a paper published in the Lancet Microbe, ${ }^{1}$ the research team found that bacterial co-infections among patients with covid-19 during the UK's first peak were rare but that antimicrobial use was high.

Antonia Ho, clinical senior lecturer and consultant in infectious diseases at the University of Glasgow, said, "Given the unprecedented challenges posed by the pandemic-particularly during its early stages when admitted patients were very sick, effective treatments were limited, and the role of possible co-infections unknown-it is unsurprising that doctors would prescribe antimicrobials.

"However, we now know that bacterial co-infection is uncommon in patients with community acquired covid-19. Since antimicrobial resistance remains one of the biggest public health challenges of our time, measures to combat it are essential to help ensure that these lifesaving medicines remain an effective treatment for infection in years to come."

\section{Stewardship interventions}

The paper included 48902 patients admitted to 260 hospitals in England, Scotland, and Wales with covid-19 between 6 February and 8 June 2020. Their average age was 74 , and $43 \%$ were female.

Before hospital admission $37 \%$ of patients (13 390 of 36145 ) had received antimicrobials in the community for that illness episode. During their hospital stay $85 \%$ (39 258 of 46061 with inpatient antimicrobial data) received at least one antimicrobial.

The researchers found that antimicrobial use was highest during the earlier stages of the first peak in March and April 2020, but it then fell during May.

Microbiological investigations, such as blood tests, were recorded in $8649(18 \%)$ of 48902 patients. Of these, $13 \%$ (1107) had covid-19 related respiratory or bloodstream bacterial infections. As a comparison, the researchers noted that bacterial co-infections occur in around $23 \%$ of patients with severe influenza.

Of the confirmed covid-19 related infections where sample timing was known, $71 \%$ (762 of 1080) were secondary, occurring more than two days after hospital admission.

Looking at the types of antimicrobials used, the researchers found that broad spectrum agents such as carbapenems-a class reserved for the treatment of severe or high risk bacterial infections-accounted for $3.8 \%$ of all prescriptions. In contrast, carbapenem alternatives were used less often, accounting for $0.2 \%-1.5 \%$ of all prescriptions.

From these findings the team concluded that a range of existing antimicrobial stewardship interventions should be prioritised for incorporation into covid-19 patient care. Suggestions included tailoring the choice of antimicrobials to likely pathogens and local resistance patterns, as well as encouraging clinicians to discontinue antimicrobials if co-infection is deemed unlikely and tests confirm that patients do not have a bacterial infection.

The study team noted some limitations to their study, including that the true rate of bacterial infections may differ from what the study reported, as only $18 \%$ of patients had microbiological investigations recorded. The team noted that microbiological diagnosis of co-infection was especially challenging during the pandemic.

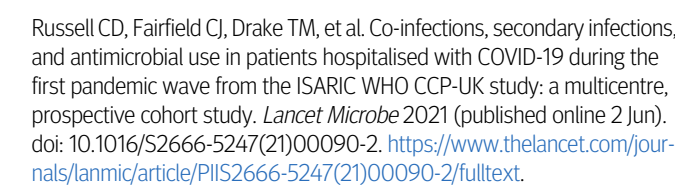

Russell CD, Fairfield C), Drake TM, et al. Co-infections, secondary infections, and antimicrobial use in patients hospitalised with COVID-19 during the first pandemic wave from the ISARIC WHO CCP-UK study: a multicentre, prospective cohort study. Lancet Microbe 2021 (published online 2 Jun). doi: 10.1016/S2666-5247(21)00090-2. https://www.thelancet.com/journals/lanmic/article/PIIS2666-5247(21)00090-2/fulltext.

This article is made freely available for use in accordance with BMJ's website terms and conditions for the duration of the covid-19 pandemic or until otherwise determined by BMJ. You may use, download and print the article for any lawful, non-commercial purpose (including text and data mining) provided that all copyright notices and trade marks are retained. 